\title{
A Preliminary Assessment of Contamination of Emergency Service Helicopters with MRSA and Multi-Resistant Staphylococcus aureus
}

\section{Sandrine Makiela, Andrew W Taylor-Robinson, Anthony Weber and Brian J Maguire*}

School of Medical \& Applied Sciences, Central Queensland University, Rockhampton, QLD, Australia

\begin{abstract}
Objective: To detect the presence of methicillin-resistant Staphylococcus aureus (MRSA) and multi-resistant $S$. aureus (Multi-RSA) in emergency service helicopters.
\end{abstract}

Methods: This proof-of-concept study used a convenience sample of two emergency service helicopters located in different Australian towns. Over a three-month period ending January 31 2015, within each helicopter five areas of perceived high contact were sampled by taking swabs on an approximately weekly basis. The precise occasions varied based on timings of cases and maintenance schedules. Swabs were analysed for the presence of MRSA Multi-RSA and other bacteria.

Results: Presumptive MRSA and other colonies were recovered from each helicopter. Of those presumptive colonies tested, $18.7 \%$ were identified as Staphylococcus aureus, $76.0 \%$ were other staphylococci (such as $S$. epidermidis), and $5.3 \%$ were other genera of bacteria. Further testing of these colonies detected no MRSA or MultiRSA.

Over the period of this study, high total numbers of bacteria were recovered in both helicopters. Typically, the floor of the helicopters had higher counts than the seat belts and the blood pressure cuff containers, whereas the radio and cardiac equipment had comparatively lower counts.

Conclusions: Since a total of $94.7 \%$ of the colonies tested were identified as Staphylococcus spp. the potential for the existence of MRSA in emergency service helicopters is apparent. Our findings highlight the importance of regular cleaning in emergency service vehicles to promote the reduction of infectious disease transmission.

We recommend the implementation of a standard operating procedure for cleaning and disinfection across all emergency service providers, reinforced by appropriate infection control training. For quality control, random intermittent swab testing of selected surfaces within all helicopters is advised. The implications of our findings may assist emergency medical service providers to reduce infectious disease transmission risks, and may help to minimise exposure to pathogenic microorganisms during disaster events including pandemics and bioterrorism.

Keywords: Emergency service care; Helicopter; Contamination; Bacteria; Staphylococcus aureus; MRSA

\section{Introduction}

Paramedics respond to almost four million calls per year in Australia [1] and 32 million calls per year in the U.S [2]. Society depends upon paramedics and other emergency medical professionals but little is known of the risks of disease transmission from contamination of vehicles, equipment or personnel. Evaluation of potential risks to patients, paramedics and to the general population must be considered in order to develop effective risk reduction interventions.

The presence of infectious disease causing microorganisms in an emergency medical environment presents potential risks to patients, friends and family members of patients, the general public, and emergency services personnel.

The detection of such organisms in emergency service vehicles illustrates the risks of these being 'vectors' of disease transmission during general operation, major incidents and disasters. Documenting these potential hazards now may promote the development of best practices which could reduce risks both on a daily basis and during large scale emergencies such as bioterrorism or pandemic.

In a country such as Australia that is sparsely populated, the provision of helicopter rescue services has become an integral part of the wider delivery of emergency medical services. This thereby makes a difference to patient management and transportation of the critically ill or seriously injured. The use of helicopters as a mode of transport has been supported in almost all regions of Australia through retrieval services such as inter-hospital/facility transport or primary response tasking to the sick and injured. The vast coverage of helicopter rescue services has been achieved through a number of public and private enterprises. These range from private charitable organizations sponsored by insurance companies that are contracted by Government departments [3], to privately run, Government-supported (crewing) of helicopter services sponsored heavily by business such as large banks [4]. Government-owned air ambulance services and community-based, privately funded enterprises contracted by state governments or state emergency service departments also provide helicopter operations [5]. [6]. Helicopter services in Australia can respond to as many as 1,200 cases per year for Government tendered operations [3] almost 1,000 operations for Government supported, sponsored services such as Westpac [4], to as low as around 300 cases per year for community based helicopter services [6].

*Corresponding author: Brian $\mathrm{J}$ Maguire, School of Medical \& Applied Sciences, Central Queensland University, Bruce Highway, Rockhampton, QLD 4702, Australia, Tel: +61749232430; E-mail: b.maguire@cqu.edu.au

Received December 22, 2015; Accepted December 30, 2015; Published January 06, 2016

Citation: Makiela S, Taylor-Robinson AW, Weber A, Maguire BJ (2016) A Preliminary Assessment of Contamination of Emergency Service Helicopters with MRSA and Multi-Resistant Staphylococcus aureus. Emergency Med 6: 304 doi:10.4172/2165-7548.1000304

Copyright: () 2016 Makiela S, et al. This is an open-access article distributed under the terms of the Creative Commons Attribution License, which permits unrestricted use, distribution, and reproduction in any medium, provided the original author and source are credited. 
The numbers of annual responses helicopter services can attend is approximately 5,000 [3]. Depending on the type of helicopter or tasking, the helicopters are usually staffed by a pilot, internal and external crewman, and a paramedic and/or doctor.

The helicopters used in this study offer services which include but are not limited to: search and rescue; primary tasking to critically ill people; inter-facility transfer (IFT) of patients; neonatal retrievals; emergency medical retrievals; and evacuation of people during disaster situations.

Although paramedics have the highest rate of occupational injury and fatality compared to other workforces in Australia [7], as well as very high injury rates internationally $[8,9]$, little is known of their risk of contracting infectious diseases in the workplace. Due to the nature of their profession, it is implicit that emergency services personnel are exposed to a range of pathogenic microorganisms including at the station, at the hospital and when out on a call. These organisms can be brought into emergency service vehicles from the environment, from patients and/or from attendants, thus providing potential for transmission to future vehicle occupants. Therefore, emergency service vehicles and their associated equipment may act as vectors for infectious organisms [10-13]. This is of particular concern regarding those bacteria that pose an increased threat due to their resistance to known antibiotics, such as the difficult-to-treat bacteria methicillinresistant Staphylococcus aureus (MRSA) and multi-resistant S. aureus (Multi-RSA).

Recent research has demonstrated a high prevalence of MRSA among emergency services personnel (6.4\%) compared to $1.5 \%$ in the general population [14]. Furthermore, previous studies have found MRSA in ambulances in both metropolitan (47.6\%) [15] and rural areas (49\%) [16]. Equipment used by emergency services personnel has also been shown to have high rates of contamination. For example, $32 \%$ of stethoscopes used by emergency services personnel tested positive for MRSA [17].

There has been very little research conducted on the microbiological contamination of emergency service helicopters. One study in the USA found large numbers of microorganisms on the interior surfaces of the vehicle [18], although the investigators did not test specifically for MRSA. To date, there has been no comparable research in Australia. Our hypothesis for this study was that emergency service helicopters are free from MRSA.

\section{Methods}

\section{Study design}

This proof-of-concept study used a convenience sample of two emergency medical helicopters located in different towns in Australia. Over a three-month period from November 12014 to January 312015 each helicopter was sampled by taking swabs on six occasions on an approximately weekly basis. The precise occasions varied based on timings of cases and maintenance schedules. The swabs were analyzed for the presence of MRSA, Multi-RSA and other bacteria, as outlined below.

\section{Experimental protocol}

At the start of this study both participating helicopter services were sent a kit containing all the materials needed to complete each swab as well as an instructional video and the packaging for return postage. At every sampling interval, the interior of each helicopter was swabbed by the onsite emergency personnel. This was performed in five areas which, following discussions with helicopter staff, were considered to have a high frequency of contact by emergency personnel and patients. These areas were: the blood pressure (BP) cuff storage bag; the buttons on the display panel of the cardiac monitor/defibrillator; the hand piece of the two-way radio; the floor surface between the emergency personnel seats and patient stretcher; and, the seat-belt buckle on the emergency personnel seats.

The swabs (transport medium swabs - Micromedia Culture Swab Plus Amies) were then transferred by courier to Central Queensland University within $24 \mathrm{~h}$ of their collection for analysis. Upon arrival, each swab was inoculated onto two Micromedia Chromogenic MRSA II agar plates and incubated at $35^{\circ} \mathrm{C}$ for $24 \mathrm{hrs}$. This medium is selective and differential for the detection of MRSA [19]. Possible MRSA colonies (referred to as presumptive colonies) appear mauve in color whereas other bacteria appear as other colours, typically blue, green or white.

After incubation, for each plate the numbers of presumptive and other colonies were counted (expressed as colony forming units). Ten percent of the presumptive colonies were selected randomly for further identification; each was subcultured onto a Micromedia Columbia Horse Blood agar plate and incubated at $35^{\circ} \mathrm{C}$ for $24 \mathrm{hrs}$. Each of the resulting cultures was tested for identification of MRSA using Queensland Medical Laboratories (QML) protocols. This involved initial testing with Staphylase test (Thermo Fisher), confirmation with tube coagulase (rabbit serum), followed by the EUCAST standardised disk diffusion method [20].

\section{Key outcome measures}

Data were also obtained from the helicopter services on the number and type of cases during the collection period. This information was correlated with the presence or absence of presumptive MRSA colonies and other bacteria, vehicle base of operation and intra-vehicle swabbed areas.

The research did not include human subjects and was therefore exempt from institutional human ethics approval.

\section{Results}

Over the three-month study period, helicopter A attended a total of 25 cases. These comprised of inter-facility transfers (IFTs) (60\%), neonatal transfers $(24 \%)$, primary responses (12\%) (including road traffic incidents and medical cases), and one search and rescue case (4\%). Helicopter B attended a total of 43 cases comprising IFTs (69\%), primary responses (30\%) (road traffic accidents, cardiac arrest and medical cases) and one case which was cancelled mid-flight (Figure 1).

Both presumptive MRSA and other colonies were recovered from each helicopter at all sampling periods, with the exception of Site B collection \#2, at which no presumptive colonies were recovered (Figure 2). Overall, the total number of colonies recovered was similar for both sites (14,399 for site A; 15,069 for site B), not including those instances where the bacteria on the plates were too numerous to count. Of the presumptive colonies tested, $18.7 \%$ were identified as Staphylococcus aureus, $76.0 \%$ were other staphylococci (such as S. epidermidis), and $5.3 \%$ were other genera of bacteria. Further testing of these colonies detected no MRSA or multi-resistant $S$. aureus.

When comparing different areas within each helicopter and/ or between successive sampling periods, there were no pronounced trends in the bacterial counts recovered (Figures 3 and 4). However, there were several indicators of possible associations. The floor of the 
helicopters typically had higher counts than the other swabbed surfaces, whereas the two-way radio and cardiac equipment had comparatively lower counts. In addition, presumptive colonies were not recovered at every sampling period, but presumptive colonies were recovered from all swabbing areas over the course of the study.

\section{Discussion}

While there was no MRSA or Multi-RSA recovered among the colonies tested, the possibility that these were present in the samples cannot be excluded. Furthermore, since $94.7 \%$ of the colonies tested were identified as Staphylococcus spp. the potential for the existence of MRSA in emergency service helicopters is very real. This is particularly likely given that the prevalence of MRSA among emergency services personnel is reported to be over four times that of the general population [14].

The high numbers of bacteria recovered in this study are comparable to the previous finding of Galtelli et al. [18]. Such a prevalence of microorganisms increases the risk of these being transferred between the vehicle, emergency services personnel, patients and their attendants. This may contribute to healthcare associated infections (HAI) as a source of "pre-hospital acquired infections." Therefore, each case has the potential to disseminate disease-causing microorganisms with the out-of-hospital transport vehicle acting as a vector.

Our results indicate no relationship between these high numbers of microorganisms and either the number or the type of cases. As shown in Figure 1, there was an increase in the number of cases during the Australian school holidays leading up to and including Christmas (collection \# 4-6), particularly in the number of primary cases, but there was no corresponding increase in microorganism counts (Figure 2). It may be inferred that this was due to the cleaning schedule and non-timetabled cleaning events. For instance, thorough cleaning of the area would have taken place soon after a body fluid spill, the occurrence of which is more likely during a primary case compared to other case types. This cleaning event, depending on thoroughness, diminishes the number of microorganisms; population growth then occurs until the surface is again sanitised.

As suggested by Figures 3 and 4, the microbial counts from floors were higher and those from the radio and cardiac equipment were lower, respectively, compared to the other swabbed surfaces. While higher numbers were expected from the floor, the lower numbers observed were in part due to the fact that in several instances there were no microbes recovered from those areas, presumably because these items were recently cleaned. This concurs with the above inference regarding non-timetabled cleaning events but may also suggest a bias based on the cleaner's perception of a given area. That is, it may be presumed that people will be more likely to clean surfaces where they expect a higher microbial count. In this context, surfaces which are customarily touched by hand, including commonly used paramedic equipment (radio and cardiac monitor/defibrillator), may be generally perceived as having higher microbial counts due to the perceived high frequency of contact by emergency services personnel and patients. The seat belts and BP cuff storage bag, which may have similar rates of contact, may not be acknowledged as such and therefore may be cleaned less frequently and/or less fastidiously, hence the comparatively higher microbial counts.

Our findings highlight the value of regular cleaning in emergency

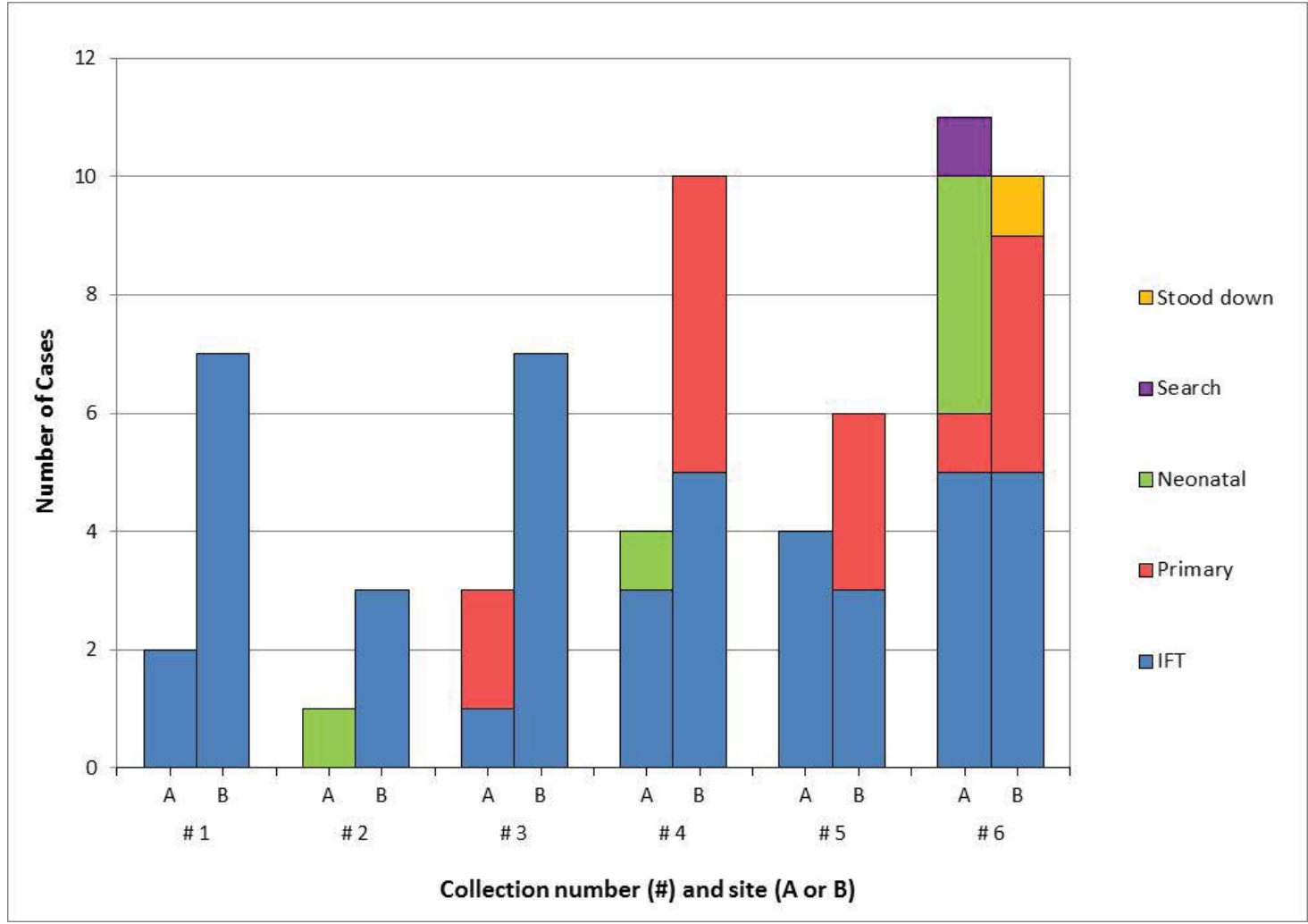

Figure 1: Number and type of emergency service helicopter cases at each site for successive microbiological sampling periods. 
Citation: Makiela S, Taylor-Robinson AW, Weber A, Maguire BJ (2016) A Preliminary Assessment of Contamination of Emergency Service Helicopters with MRSA and Multi-Resistant Staphylococcus aureus. Emergency Med 6: 304. doi:10.4172/2165-7548.1000304

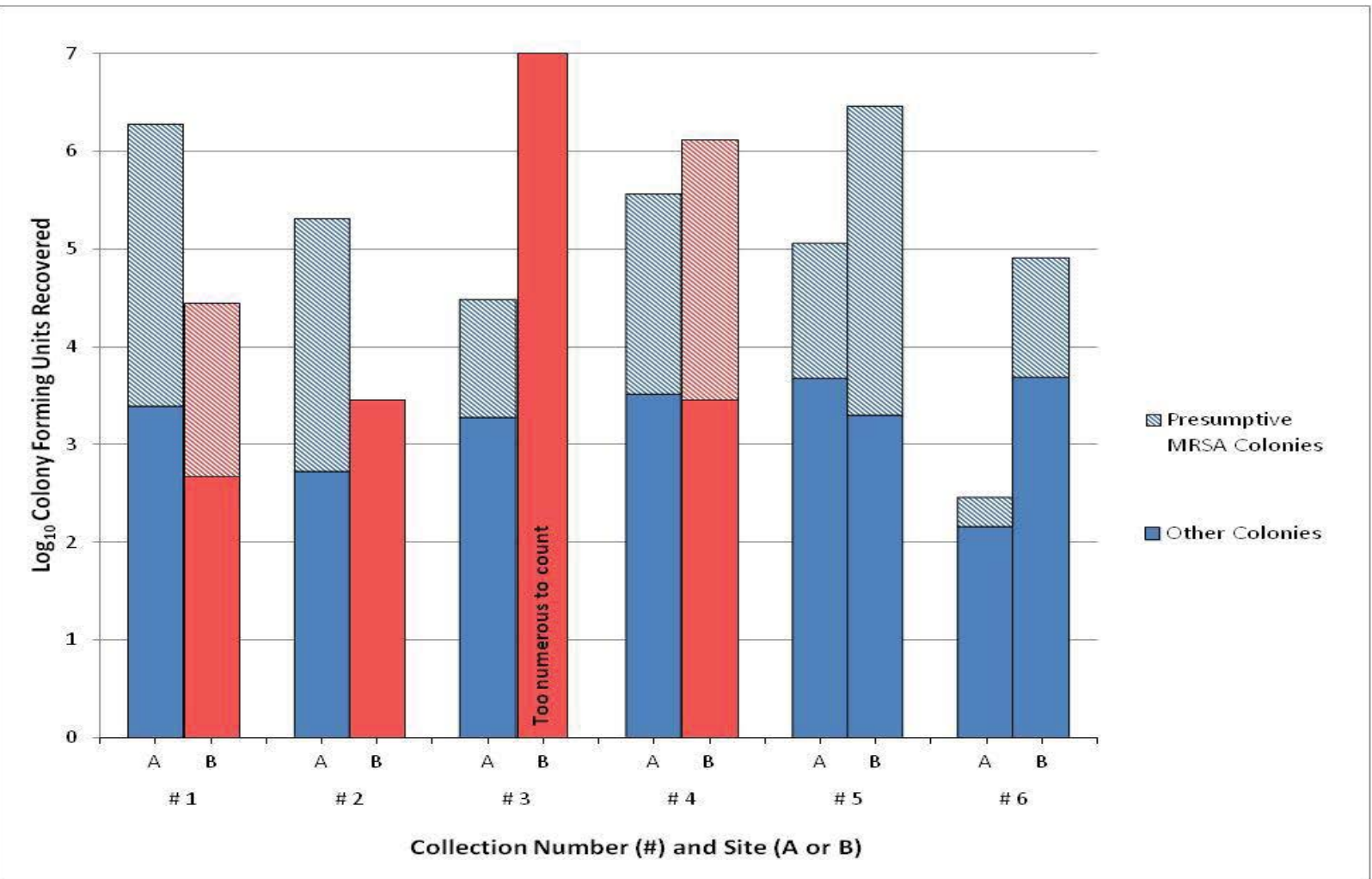

Figure 2: Number and type of bacterial colonies recovered at each emergency service helicopter site for successive microbiological sampling periods. Bacterial counts are presented as $\log _{10}$ of colony forming units.

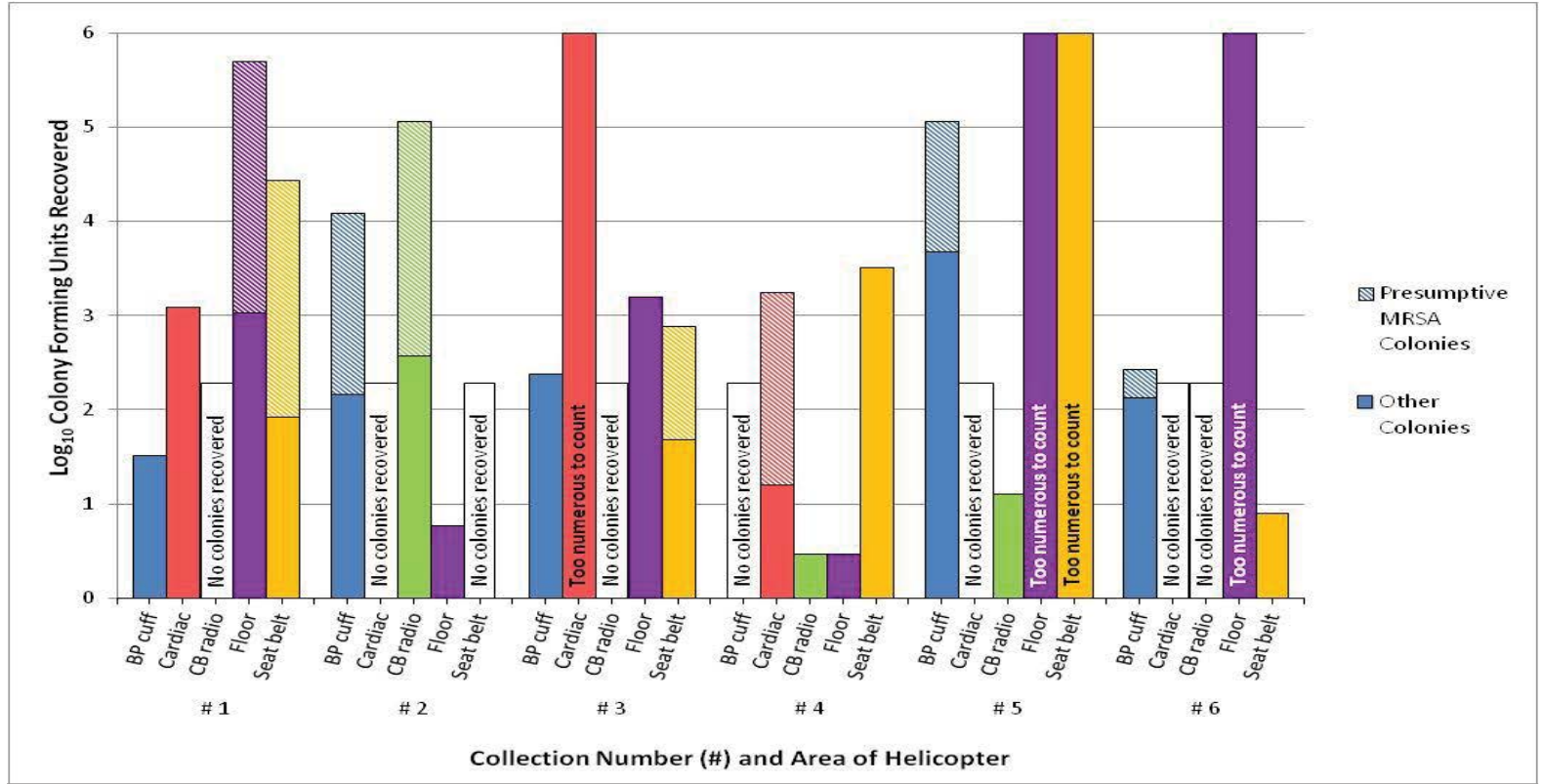

Figure 3: Number and type of bacterial colonies recovered from different internal areas of the helicopter at emergency service site A for successive microbiological sampling periods. Bacterial counts are presented as $\log _{10}$ of colony forming units. 


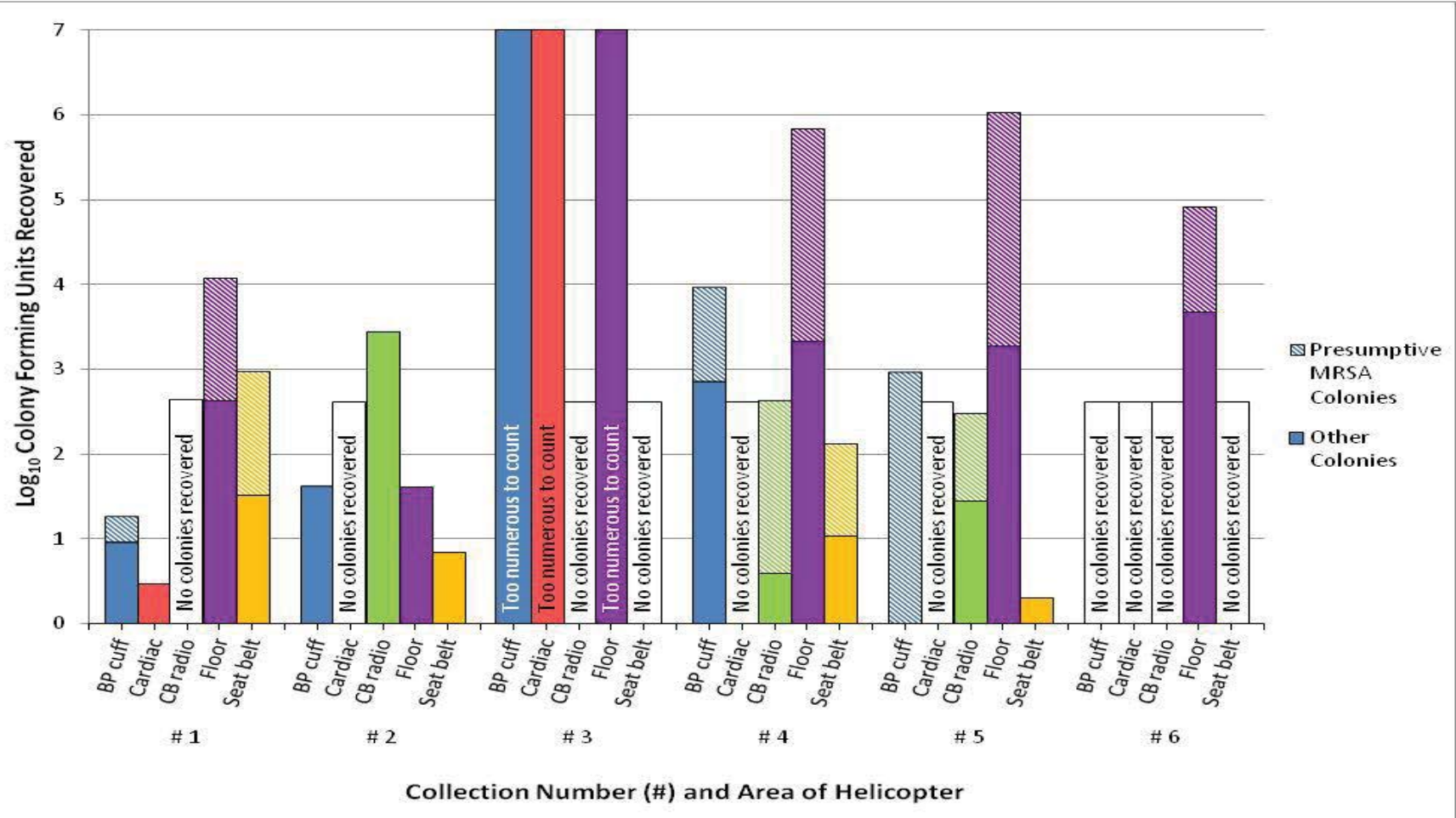

Figure 4: Number and type of bacterial colonies recovered from different internal areas of the helicopter at emergency service site B for successive microbiological sampling periods. Bacterial counts are presented as $\log _{10}$ of colony forming units.

service vehicles to aid the prevention of disease transmission. Attempts have been made to reduce disease transmission through the use of new, supposedly antimicrobial textiles [21]; these fabrics have been incorporated into the manufacture of uniforms for emergency services personnel [22]. However, Groß et al. tested one such fabric designed to reduce contamination risks and found no significant difference in microbial contamination compared to standard materials [23]. Nevertheless, as most of the surfaces in an emergency service vehicle are non-porous, including those swabbed in this study, microbial growth is not intrinsically encouraged. Growth may occur if those surfaces are soiled with organic matter of any origin. This includes sweat and other skin secretions which are not as easily visible as other bodily fluids. Again, this stresses the paramount importance of routine and meticulous cleaning protocols.

We found no standard policies or standard operating procedures pertaining to cleaning and disinfection of emergency service helicopters across Australia. This contrasts with the availability of guidelines for other types of out-of-hospital transport vehicle, such as ambulances [24]. Generic federal guidelines do exist for the prevention and control of infection, providing a basis for all healthcare professions to develop specific protocols and processes tailored to their individual settings [25]. As a consequence, protocols vary from state to state and between service providers, creating different levels of risk of disease transmission within the same industry. There are also variations in protocol implementation including training and day to day operations. In addition, it is likely that protocols are adhered to less stringently during periods of unusually high case loads or in natural disaster scenarios such as disease epidemics, pandemics and bioterrorism events. While this is understandable, it may lead to an increased risk of disease transmission, thereby exacerbating these crises.
This pilot study highlights the fact that there are high numbers of potentially disease-causing microorganisms in emergency service helicopters. Based on these results, and with the health and safety of helicopter emergency services personnel and patients in mind, it is our recommendation that all providers across Australia develop and adopt standard operation procedures with respect to cleaning and disinfection of emergency services vehicles. This would include a regular cleaning schedule for the aircraft, vehicles and associated equipment, preferably after every shift. In addition, as is current practice during a shift, cleaning should also be undertaken as soon as is practicable after a body fluid spill or other contamination. Ideally, all cleaning would be performed by a specialist team rather than by the crew, as is done with ambulances in England [26]. As a quality assurance measure, we recommend random swab testing of selected surfaces within all emergency services vehicles, performed several times per annum.

Further recommendations would include the implementation of a standard induction for new staff in microbiological health and safety in the workplace, and regular in-house refresher courses in infectioncontrol procedures. Microbiological education should also form an integral part of university undergraduate courses in paramedic science, as already offered at some higher education establishments [27], thereby providing graduates with a prerequisite knowledge of infection control.

It is acknowledged that despite these measures, emergency services personnel are not always aware of the infection status of patients, particularly with primary cases. Under such circumstances, feedback from hospitals on patient diagnosis may better inform required cleaning procedures. In an era in which case recording is predominantly online, information sharing of this kind should be straightforward to facilitate. 
However, since even the best information sharing is sometimes delayed, emergency services personnel should generally assume the worst and protect and disinfect accordingly.

The aforementioned recommendations could extend to all emergency service vehicles and personnel, particularly if future studies were to be broadened to include these. Further research may encompass a larger sample size, more frequent sampling and the provision of information on all cleaning events. The efficacy and efficiency of different cleaning products and procedures should also be determined.

\section{Conclusions}

This study showed that MRSA was not recovered from the emergency service helicopters examined, but did indicate the potential for the presence of these antibiotic resistant bacteria. The large numbers of microorganisms recovered demonstrate the necessity for standardized cleaning protocols as well as appropriate staff training for their implementation. Such measures would thereby reduce the risk of infectious disease transmission in the emergency services profession and ensure a cleaner, safer medical environment that demonstrates industry best practice. The implications of our findings may help emergency medical organizations to save lives, speed recovery and serve the community by enabling the provision of the highest standards of rapid response critical care. Enabling these standards on a day-today basis may also help emergency service organizations be better prepared for large-scale infectious disease transmission events such as pandemics or bioterrorism.

\section{Acknowledgements}

This study received financial support from Central Queensland University's Research Development \& Incentives Program; Merit Grant number 0980022829 "Antibiotic-resistant bacterial contamination of emergency medical vehicles - is there a risk to patients and providers?" We thank Olivia Daniels for microbiology laboratory assistance.

\section{Declaration of Interest}

The authors report no conflicts of interest. The authors alone are responsible for the content and writing of the paper.

\section{References}

\section{References}

1. Australian Government Productivity Commission. Report on Government Services. 2013. http://www.pc.gov.au/ data/assets/pdf file/0019/121771/13government-services-2013-chapter9.pdf

2. Maguire BJ, Walz BJ (2004) Current Emergency Medical Services Workforce Issues in the United States. Journal of Emergency Management 2:17-26.

3. Careflight. History of Careflight 2015. http://careflight.org/about/history1/.

4. Westpac Rescue Helicopter Service. History of Westpac Rescue. 2015. http:// www.rescuehelicopter.com.au/About/History.

5. Queensland Government. Rescue Services in Queensland 2013. https://www. qld.gov.au/emergency/emergencies-services/rescue-services.html.

6. RACQ Capricorn Helicopter Rescue Service. Mission Statement 2015. https:// chrs.org.au/.

7. Maguire BJ, O'Meara P, Brightwell R, O'Neill BJ, FitzGerald G (2014) Occupational injury risk among Australian paramedics: an analysis of national data. Med J Aust 200: 477-480.
8. Maguire BJ, Hunting KL, Guidotti TL, Smith GS (2005) Occupational injuries among emergency medical services personnel. Prehosp Emerg Care 9: 405411.

9. Maguire BJ, Smith S (2013) Injuries and fatalities among emergency medical technicians and paramedics in the United States. Prehosp Disaster Med 28 : 376-382.

10. Nigam Y, Cutter J (2003) A preliminary investigation into bacterial contamination of Welsh emergency ambulances. Emerg Med J 20: 479-482.

11. Eibicht SJ, Vogel U (2011) Meticillin-resistant Staphylococcus aureus (MRSA) contamination of ambulance cars after short term transport of MRSA-colonised patients is restricted to the stretcher. J Hosp Infect 78: 221-225.

12. Alves DW, Bissell RA (2008) Bacterial pathogens in ambulances: results of unannounced sample collection. Prehosp Emerg Care 12: 218-224.

13. Yoo IS, You YH, Kwon KC, Jeong TO (2012) Bacterial contamination conditions in ambulances and their equipment in South Korea. Journal of The Korean Society of Emergency Medicine. 23: 1-7.

14. Amiry AA, Bissell RA, Maguire BJ, Alves DW (2013) Methicillin-resistant Staphylococcus aureus nasal colonization prevalence among emergency medical services personnel. Prehosp Disaster Med 28: 1-5.

15. Roline CE, Crumpecker C, Dunn TM (2007) Can methicillin-resistant Staphylococcus aureus be found in an ambulance fleet? Prehosp Emerg Care 11: $241-244$.

16. Brown R, Minnon J, Schneider S, Vaughn J (2010) Prevalence of methicillinresistant Staphylococcus aureus in ambulances in southern Maine. Prehosp Emerg Care 14: 176-181.

17. Merlin MA, Wong ML, Pryor PW, Rynn K, Marques-Baptista A, et al. (2009) Prevalence of methicillin-resistant Staphylococcus aureus on the stethoscopes of emergency medical services providers. Prehosp Emerg Care 13: 71-74.

18. Galtelli M, Deschamp C, Rogers J (2006) An assessment of the prevalence of pathogenic microorganisms in the rotor wing air ambulance: one program's findings. Air Med J 25: 81-84.

19. Yang HY, Suh JT, Lee HJ (2010) Evaluation of commercial selective agars in screening for methicillin-resistant Staphylococcus aureus. Ann Clin Lab Sci 40: 252-256.

20. The European Committee on Antimicrobial Susceptibility Testing. Breakpoint Tables for Interpretation of MICs and Zone Diameters. 2014. http://www. eucast.org.

21. Gao Y, Cranston $R$ (2008) Recent advances in antimicrobial treatments of textiles. Textile Research Journal 78: 60-72.

22. O'Hanlon SJ, Enright MC (2009) A novel bactericidal fabric coating with potent in vitro activity against meticillin-resistant Staphylococcus aureus (MRSA). Int J Antimicrob Agents 33: 427-431.

23. Groß R, Hübner N, Assadian O, Jibson B, Kramer A (2010) Pilot study on the microbial contamination of conventional vs. silver-impregnated uniforms worn by ambulance personnel during one week of emergency medical service. GMS Krankenhhyg Interdiszip.

24. Queensland Ambulance Service. Infection Control Framework. Version 1 effective 7 August 2014. https://ambulance.qld.gov.au/docs/.

25. National Health and Medical Research Council. Australian Guidelines for the Prevention and Control of Infection in Healthcare. 2010. https://www.nhmrc. gov.au.

26. National Patient Safety Agency. The National Specifications for Cleanliness in the NHS: a Framework for Setting and Measuring Performance Outcomes in Ambulance Trusts. 2008. http://www.nrls.npsa.nhs.uk/ resources/?Entryld45=61467

27. Central Queensland University. CG95 Bachelor of Paramedic Science 2015 https://handbook.cqu.edu.au/ programs/index?programCode=CG95. 